\title{
An Unusual Cause of Upper Gastrointestinal Bleeding: Duodenal Vascular Ectasia after Splenectomy
}

\author{
Kamil Ozdil, Abdurrahman Sahin, Resul Kahraman, Turan Calhan, Recai Gokcan, \\ Murat Hakan Karabulut, Haci Mehmet Sokmen
}

\begin{abstract}
Cavernomatous transformation of portal vein (CTPV) is characterized by a collateral network of veins in the hepatic hilum that bypasses an obstructed or occluded extrahepatic portal vein. We report a case of CTPV, 25 years after splenectomy in a 39-year-old man with acute and chronic blood loss from duodenal vascular ectasia without esophageal or gastric varices or portal hypertensive gastropathy. This case demonstrates that distal part of duodenum assessment is important in gastrointestinal bleeding of patients with CTPV.
\end{abstract}

Abbreviations: GI: Gastrointestinal; CTPV: Cavernomatous transformation of portal vein; CT: Computed tomography; PHD: Portal hypertensive duodenopathy.

Keywords: Cavernomatous transformation of portal vein, Gastrointestinal bleeding, Splenectomy, Vascular ectasia.

How to cite this article: Ozdil K, Sahin A, Kahraman R, Calhan T, Gokcan R, Karabulut MH, Sokmen HM. An Unusual Cause of Upper Gastrointestinal Bleeding: Duodenal Vascular Ectasia after Splenectomy. Euroasian J Hepato-Gastroenterol 2012;2(2):109-112.

Source of support: Nil

Conflict of interest: None

\section{INTRODUCTION}

Duodenal vascular ectasia is a rare cause of upper gastrointestinal (GI) bleeding. ${ }^{1}$ It is associated with endstage renal disease and aortic valve diseases, as well as a component of portal hypertension. ${ }^{1,2}$ Portal/splenic vein thrombosis, development of portal hypertension and portal vein cavernomatous transformation may be seen after splenectomy. ${ }^{3}$ Cavernomatous transformation of portal vein (CTPV) is new vascular network formation around gall bladder, intrahepatic and extrahepatic biliary tract as a result of portal vein obstruction. ${ }^{4}$ We presented a case with CTPV extending to duodenum while there were neither esophageal varices nor gastric varices.

\section{CASE REPORT}

A 39-year-old man with history of splenectomy and distal pancreatectomy because of a traffic accident 25 years ago was admitted to the hospital with the symptoms of fatigue, palpitation and intermittent melena for 3 months. He had a history of diabetes for 3 years, but no history of liver disease or alcohol abuse. On physical examination, he was pale; chest and heart examinations were normal. The abdomen was soft and there was no abdominal mass or bruit. Laboratory data showed that hemoglobin concentration was $8.6 \mathrm{gm} / \mathrm{dl}$, erythrocyte count was $4.11 \mathrm{million} / \mathrm{mm}^{3}$, platelet count $434,000 / \mathrm{mm}^{3}$, alanine aminotransferase was 25 (2055) U/l, aspartate aminotransferase was $24 \mathrm{U} / \mathrm{l}$, gammaglucronyl transferase was 133 (53-150) IU/l, alkaline phosphatase was 139 (102-450) U/l, albumin was 4.7 (3.44.2) $\mathrm{gm} / \mathrm{dl}$, serum iron was $24(32-283) \mu \mathrm{g} / \mathrm{dl}$. On esophagogastroduodenoscopy, there were no esophageal or gastric varices but in the second part of duodenum vascular ectasia lesions was detected (Fig. 1). Endoscopic biopsy was performed on vascular ectatic mucosa and pathologic examination revealed focal lymphangiectasia and grossly dilates blood vessels between the muscularis mucosal and submucosal layers (Fig. 2). Local hemostatic agent was used to achieve hemostasis after biopsy and bleeding stopped without any complications. Dynamic computed tomography (CT) showed liver steatosis and CT angiography revealed CTPV and varicose vascular lesions around the duodenum connected with portal vein (Figs 3A to D). The patient was stabilized with supportive therapy. He was discharged 1 week after admission with propranolol prophylaxis. At 3-month follow-up, clinical assessment and laboratory data did not show any evidence of bleeding.

\section{DISCUSSION}

Noncirrhotic portal hypertension causes upper GI bleeding via esophageal or gastric varices and portal hypertensive

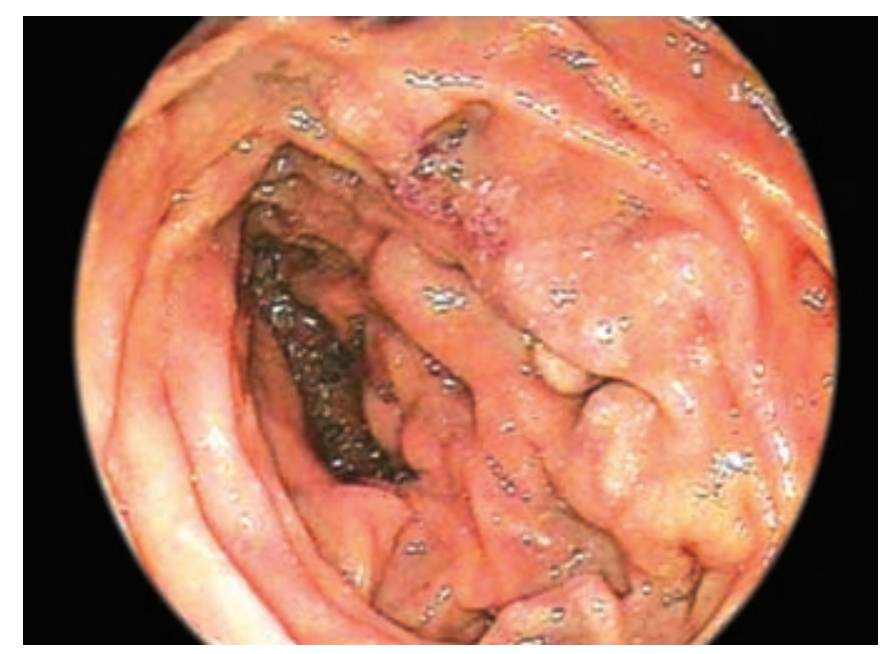

Fig. 1: Esophagogastroduodenoscopy of the patient 


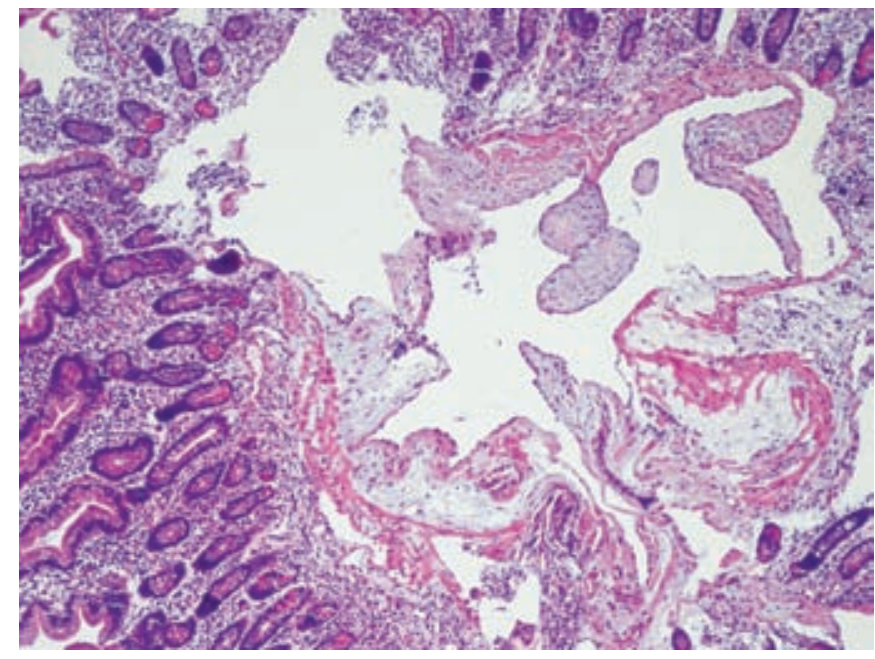

Fig. 2: Biopsy findings of vascular ectatic mucosa

gastropathy/enteropathy development. ${ }^{5}$ Portal/splenic vein thrombosis occur between 0.35 and $19 \%$ after splenectomy. ${ }^{3}$ Splenic vein thrombosis leads to a localized, left-sided or
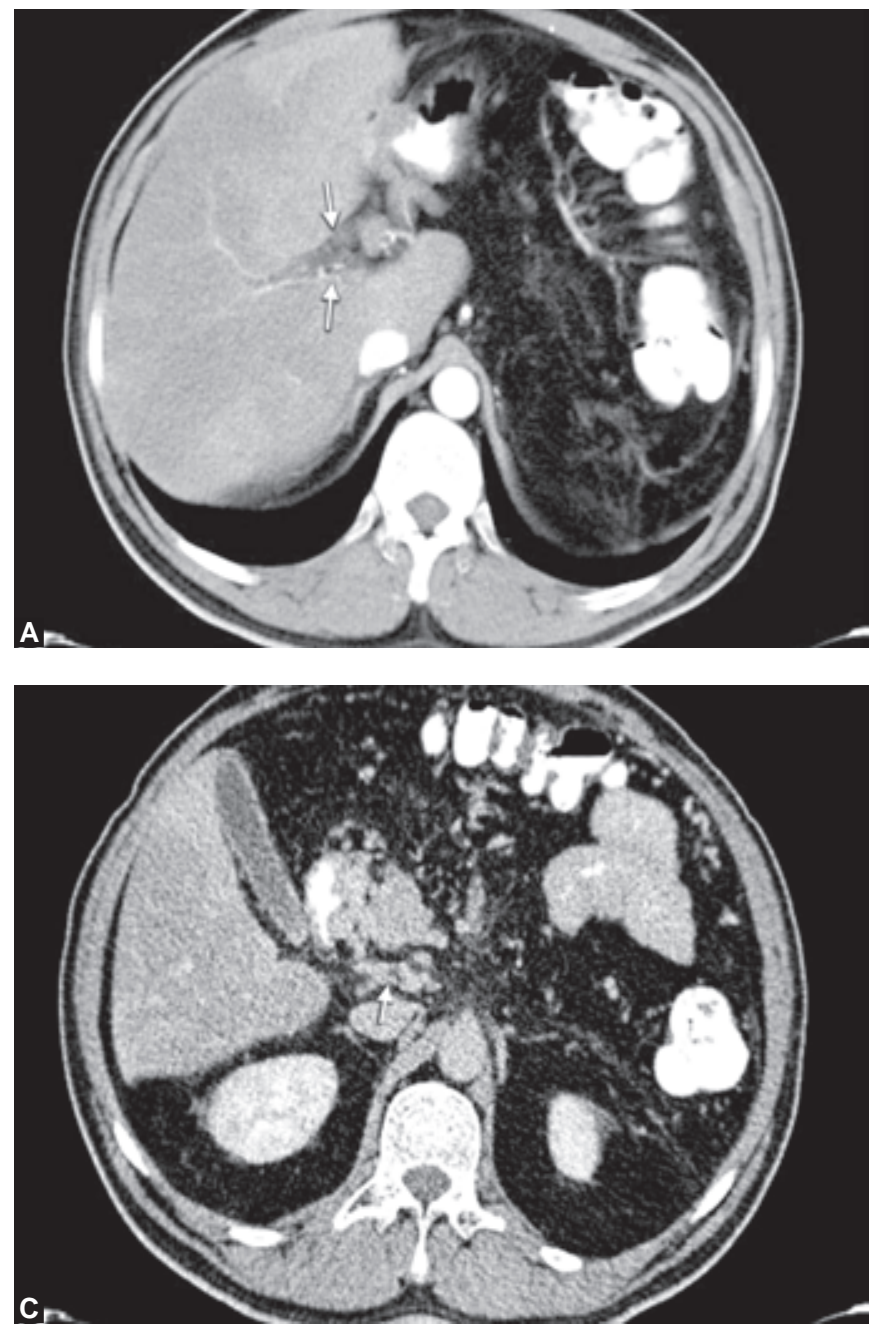

sinistral hypertension. Pancreatic surgery is another cause of splenic vein thrombosis. ${ }^{6}$ Isolated gastric varices result from thrombosis or obstruction of the splenic vein resulting in back pressure changes in the left portal system. The patient in this case report did not present with gastric varices or portal hypertensive gastropathy. CT angiography revealed only CTPV without splenic or portal vein thrombosis.

Cavernous transformation of the portal vein (also called portal cavernoma) in adults is an uncommon finding. In the pediatric age group, it is frequently associated to congenital anomalies. After thrombosis of the portal vein, in the absence of liver disease, organization and subsequent recanalization of the thrombus take place, followed by the development of hepatopedal portal collateral vessels, in order to restore portal perfusion, thus leading to the formation of a typical CTPV. ${ }^{7}$ The formation of these vessels is necessary in order to drain splenic and mesenteric venous flow into the intrahepatric portal branches and to maintain sufficient hepatic blood flow and normal hepatic function.
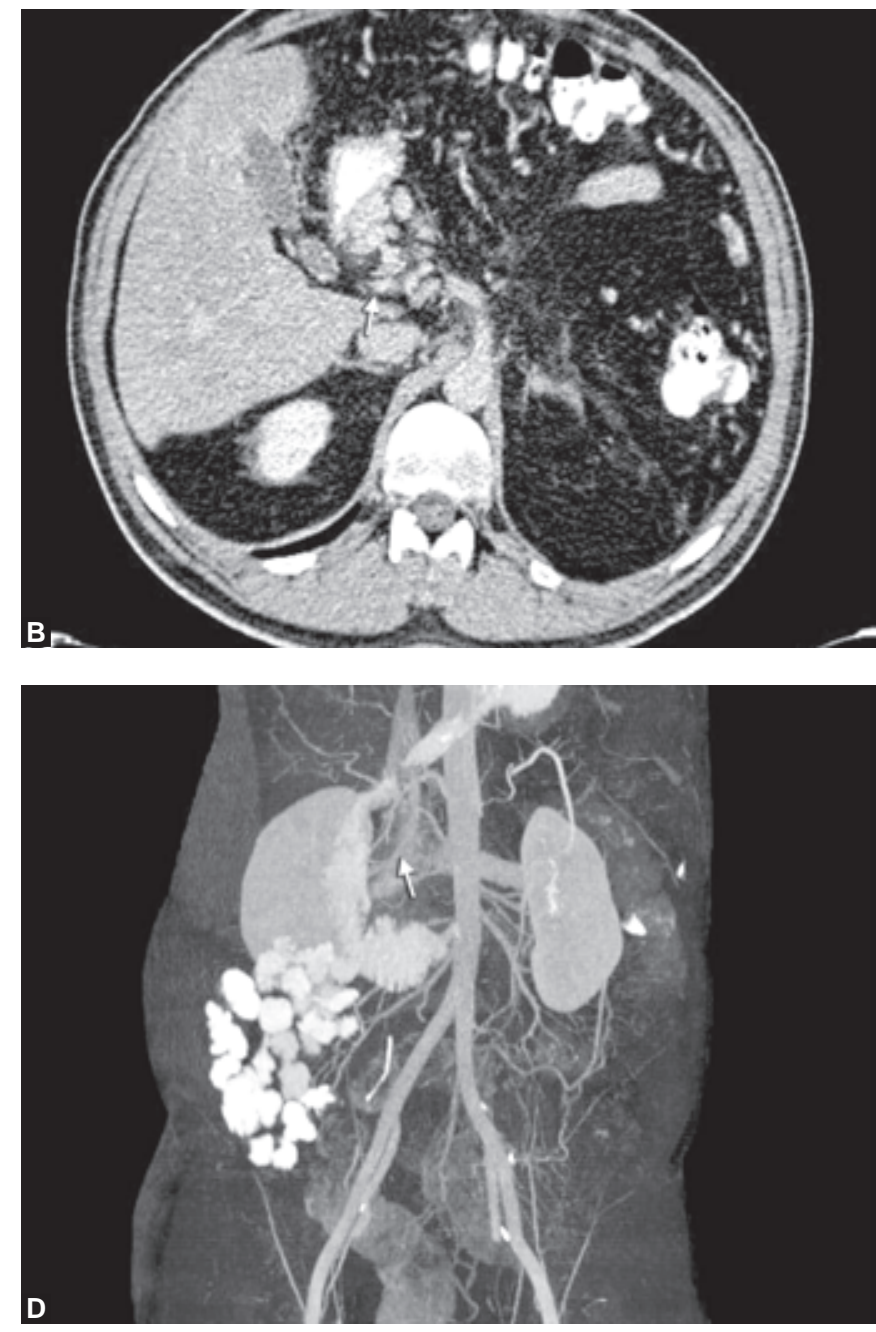

Figs 3A to D: Dynamic CT showing hepatic steatosis and varicose vascular lesions around the duodenum 
These hepatopedal collateral vessels look like cavernous angioma when observed in gross specimen sections. CTPV usually presents with upper GI bleeding due to esophageal varices. CTPV may presents only with jaundice, due to compression of the common bile duct. In some cases, CTPV can favor the development of biliopathy. Whether the formation of portal thrombosis results in typical CTPV depends on the duration of portal obstruction. In our patient, detection of CTPV suggested the development of portal vein thrombosis after surgery. When portal obstruction is limited to the portal trunk only, the hepatopedal collateral veins of CTPV may skip the obstructed site and connect the intralobular open portal branch to maintain normal portal perfusion. In cases where portal obstruction is more extensive, even though the hepatopedal collateral veins of CTPV may take part in portal circulation, they are not sufficient to relieve portal hypertension, thus causing esophageal varices and formation of portacaval collateral circulation. ${ }^{4}$ Changes in intra-abdominal pressure during splenectomy decrease portal vein blood flow and induce stasis. Both stasis of venous flow and resulting congested coagulation factors may induce portal vein thrombosis. Stasis of blood in the stump of the splenic vein might be another mechanical factor for portal vein thrombosis. Turbulence in the stump of the splenic vein may result thrombosis and thrombus spread into portal vein. Although platelet count and hypercoagulability can increase postoperatively, association between these factors with formation of thrombosis at portal system is unclear. Thrombosis at portal system may be influenced by numerous technical factors, such as early ligation of the splenic artery, use of the endoscopic vascular stapler, or distal or proximal ligation of the splenic vein, but their definitive role is controversial. ${ }^{8,9}$ Interestingly, in this case, the extension of collateral veins to duodenum without varices suggested that portal obstruction was limited and collateral vessels at duodenum might be an anatomical variation or a consequence of technical factors at surgery.

Only a few cases of vascular ectasia of the duodenum have been reported in patients with cirrhosis who had esophageal varices or portal hypertensive gastropathy. ${ }^{1}$ Duodenal lesions in portal hypertensive patients are clinically significant because of being a possible source of massive bleeding. Barakat et al found portal hypertensive duodenopathy (PHD) in $51.4 \%$ of cirrhotic patients. Moreover, study have shown that PHD restricted to second part in duodenum was only $3.8 \%$ within PHD group and none of them had duodenal vascular ectasia. ${ }^{2}$ In the present case, there was bleeding from varicous collateral veins which originated from portal vein and extended to duodenum in the absence of portal hypertension findings at esophagus or stomach. To our knowledge, this is the first patient who has bleeding from isolated duodenal vascular ectasia with CTPV and noncirrhotic portal hypertension.

Consequently, this case highlights the point that CTPV should be suspected in patients who had a history of splenectomy. Isolated duodenal vascular ectasia should be kept in mind as a rare cause of upper GI bleeding.

\section{REFERENCES}

1. Lee BJ, Park JJ, Seo YS, et al. Upper gastrointestinal bleeding from duodenal vascular ectasia in a patient with cirrhosis. World J Gastroenterol 2007;13:5154-57.

2. Barakat M, Mostafa M, Mahran Z, Soliman AG. Portal hypertensive duodenopathy: Clinical, endoscopic and histopathologic profiles. Am J Gastroenterol 2007;102:2793-802.

3. Krauth MT, Lechner K, Neugebauer EA, Pabinger I. The postoperative splenic/portal vein thrombosis after splenectomy and its prevention—an unresolved issue. Haematologica 2008; 93:1227-32.

4. Wang L, Li ZS, Lu JP, Wang F, Liu Q, Tian JM. Cavernous transformation of the portal vein: Three-dimensional dynamic contrast-enhanced MR angiography. Abdom Imaging 2008;33: 463-68.

5. Cichoz-Lach H, Celiñski K, Slomka M, Kasztelan-Szczerbiñska B. Pathophysiology of portal hypertension. J Physiol Pharmacol 2008;59(Suppl)2:231-38.

6. Köklü S, Coban S, Yüksel O, Arhan M. Left-sided portal hypertension. Dig Dis Sci 2007;52:1141-49.

7. Yonem $\mathrm{O}$, Bayraktar $Y$. Is portal vein cavernous transformation a component of congenital hepatic fibrosis? World J Gastroenterol 2007;13:1928-29.

8. Targarona EM. Portal vein thrombosis after laparoscopic splenectomy: The size of the risk. Surg Innov 2008;15:266-70.

9. van’t Riet M, Burger JW, van Muiswinkel JM, Kazemier G, Schipperus MR, Bonjer HJ. Diagnosis and treatment of portal vein thrombosis following splenectomy. Br J Surg 2000;87: 1229-33.

\section{ABOUT THE AUTHORS}

\section{Kamil Ozdil (Corresponding Author)}

Department of Gastroenterology, Umraniye Training and Research Hospital, Adem Yavuz Cad No. 1 Umranyie, Istanbul, Turkey e-mail: kamilozdil@gmail.com

\section{Abdurrahman Sahin}

Department of Gastroenterology, Umraniye Training and Research Hospital, Istanbul, Turkey

\section{Resul Kahraman}

Department of Gastroenterology, Umraniye Training and Research Hospital, Istanbul, Turkey 


\section{Turan Calhan}

Department of Gastroenterology, Umraniye Training and Research Hospital, Istanbul, Turkey

\section{Recai Gokcan}

Department of Gastroenterology, Umraniye Training and Research Hospital, Istanbul, Turkey

\section{Murat Hakan Karabulut}

Department of Gastroenterology, Umraniye Training and Research Hospital, Istanbul, Turkey

\section{Haci Mehmet Sokmen}

Department of Gastroenterology, Umraniye Training and Research Hospital, Istanbul, Turkey 